\title{
Evaluating GPS biologging technology for studying spatial ecology of large constricting snakes
}

\author{
Brian J. Smith ${ }^{{ }^{*}}\left(\mathbb{0}\right.$, Kristen M. Hart ${ }^{2}$, Frank J. Mazzotti ${ }^{3}$, Mathieu Basille ${ }^{3}$ (i) and Christina M. Romagosa ${ }^{4}$ (D)
}

\begin{abstract}
Background: GPS telemetry has revolutionized the study of animal spatial ecology in the last two decades. Until recently, it has mainly been deployed on large mammals and birds, but the technology is rapidly becoming miniaturized, and applications in diverse taxa are becoming possible. Large constricting snakes are top predators in their ecosystems, and accordingly they are often a management priority, whether their populations are threatened or invasive. Fine-scale GPS tracking datasets could greatly improve our ability to understand and manage these snakes, but the ability of this new technology to deliver high-quality data in this system is unproven. In order to evaluate GPS technology in large constrictors, we GPS-tagged 13 Burmese pythons (Python bivittatus) in Everglades National Park and deployed an additional 7 GPS tags on stationary platforms to evaluate habitat-driven biases in GPS locations. Both python and test platform GPS tags were programmed to attempt a GPS fix every 90 min.

Results: While overall fix rates for the tagged pythons were low (18.1\%), we were still able to obtain an average of 14.5 locations/animal/week, a large improvement over once-weekly VHF tracking. We found overall accuracy and precision to be very good (mean accuracy $=7.3 \mathrm{~m}$, mean precision $=12.9 \mathrm{~m}$ ), but a very few imprecise locations were still recorded $(0.2 \%$ of locations with precision $>1.0 \mathrm{~km})$. We found that dense vegetation did decrease fix rate, but we concluded that the low observed fix rate was also due to python microhabitat selection underground or underwater. Half of our recovered pythons were either missing their tag or the tag had malfunctioned, resulting in no data being recovered.

Conclusions: GPS biologging technology is a promising tool for obtaining frequent, accurate, and precise locations of large constricting snakes. We recommend future studies couple GPS telemetry with frequent VHF locations in order to reduce bias and limit the impact of catastrophic failures on data collection, and we recommend improvements to GPS tag design to lessen the frequency of these failures.
\end{abstract}

Keywords: GPS telemetry, Biologging, Habitat bias, Large constricting snakes, Invasive species

\section{Background}

Biotelemetry has the potential to bring ecologists the data needed both to address questions of ecological theory and to improve conservation and management strategies [1-3]. VHF radio tracking was first used on animals in the 1960s and has allowed researchers to study the movement of elusive animals [4]. Other satellite-based technologies have emerged since then (e.g., ARGOS),

\footnotetext{
*Correspondence: bjsmith@usgs.gov

${ }^{1}$ Cherokee Nation Technologies, Davie, FL, USA

Full list of author information is available at the end of the article
}

but GPS technology, in particular, has revolutionized our understanding of animal movement by providing relatively accurate, frequent locations throughout the day and in conditions that previously hampered tracking [5]. For example, endangered Florida panthers (Puma concolor coryi) were thought to have selected only forested habitats when data were collected primarily in the daytime with VHF radiotelemetry. Further investigation of panther habitat selection using GPS tracking throughout the entire day confirmed the importance of forested 
habitat, but also showed the selection of open habitats as hunting grounds at night, a novel insight [6].

While GPS does represent a major technological advancement, it still comes with potential drawbacks, including larger tag size (compared with a VHF transmitter), high cost (and thus a trade-off with low sample size of individuals), low positional precision (compared to direct observation, although superior to many alternative technologies), and habitat-driven location bias [7-9]. Given these potential hurdles, deciding whether or not GPS is the right tool for tackling relevant ecological or management questions is an important consideration [10]. Compared with VHF tracking, GPS tracking is generally a superior choice for biological questions that require more locations per animal (as opposed to more animals in the population) or for biological questions focused on temporally fine-scale movements [4].

GPS tagging has typically been limited to those species large enough to carry a heavy device-until recently this was only large mammals and large birds-and thus many of the assumptions of GPS performance (e.g., fix rate, habitat-driven variance in fix rate) are based on large units deployed on these taxa [11]. To study reptiles, GPS biotelemetry (as opposed to satellite tracking, e.g., the ARGOS system, for definitions see [3]) has been used only sparingly, primarily on marine turtles [e.g., 12] and crocodilians [e.g., 13-15]. Among squamates, only large lizards such as blue-tongue skinks and monitor lizards have been tracked using GPS tags [e.g., 16, 17]. Hart et al. [18] reported on the first preliminary application of GPS technology in a large constrictor, the Burmese python (Python bivitattus). This was the first documented GPS application in any snake.

Large constrictors are ecologically important and historically understudied [19]. Many large constrictors are considered threatened or declining in their native range [20-22], whereas other species have become invasive [23], and management of both invasive and imperiled large constrictors would benefit from an improved understanding of their spatial ecology. VHF telemetry has been used to study large constrictor behavior and ecology around the world, including studies on a variety of taxa (e.g., pythons, boas, and anacondas) in a number of different countries (e.g., Argentina, Australia, Bangladesh, South Africa, USA, and Venezuela) [18, 24-30]. Many of these VHF studies yielded infrequent, irregularly timed, and predominantly daytime locations, and increasing the frequency and regularity of VHF locations in studies of snakes is often difficult due to logistical constraints (e.g., long time to manually record a VHF fix, workload, site inaccessibility, safety-particularly when tracking at night, etc.) [28]. GPS has the potential to provide these data and allow for finer-scale investigation of spatial patterns; however, there are a number of challenges to tracking large constrictors with GPS tags. Telemetry on snakes almost always involves surgical implantation of a tag [e.g., 31], since snakes cannot wear collars [28]. GPS signal attenuates under the skin, and therefore, these tags must have an external antenna that exits through the skin, potentially compromising the animal's health and tag retention. Moreover, many large constrictors have been shown to select habitats with thick vegetation cover $[18,20,32,33]$, which is likely to reduce GPS fix rates [8]. Furthermore, some large constrictors often spend time in water and can tolerate high salinities [34], further increasing the likelihood of signal loss [5].

\section{Methods \\ Aim}

We explored whether GPS technology could provide the data necessary to answer questions about large constrictor spatial ecology that we have been unable to answer with VHF telemetry. Specifically, we aimed to determine whether GPS technology could provide an increased number of locations/animal/week without sacrificing accuracy and precision.

\section{Study system}

Our study system was the invasive population of Burmese pythons in Everglades National Park (ENP), Florida, USA. Burmese pythons have likely been established and breeding in ENP since the mid-1980s [35]. Their remarkable crypsis makes them impossible to study by direct observation [36], so most of what we know about their movement patterns and habitat selection is based on VHF radio tracking in ENP [18, 32, 37]. Due to the logistical difficulties of working in the vast Everglades landscape, VHF tracking has typically been limited to one location/animal/week, obtained during daylight hours, severely limiting the inferences that can be drawn about python spatial ecology. Most research has focused on larger-scale questions about home range size and population-level habitat use, but no research to date has focused on fine-scale movement patterns or individual variation. Burmese pythons are an ideal snake for this GPS tagging endeavor. First, their large body size can accommodate a larger tag than most snakes could accommodate. Second, while animal care is still a top priority, the risk of potentially needing to euthanize an invasive Burmese python due to health concerns is more acceptable than the risk of potentially needing to euthanize a native species of conservation concern. Third, their striking impacts to the mammal community in ENP make them a top research and management priority [38-40]. 


\section{Field methods}

\section{Python captures}

All potential study animals were captured along the main roads in ENP, including State Road 9336 (Main Park Road), Research Road, and Old Ingraham Highway. Most animals were located by road cruising from a vehicle, although some searchers chose to walk sections of road such as Old Ingraham Highway. They were captured by hand either by a researcher or by a permitted volunteerin-park (VIP) or intern in ENP.

In order to be considered for GPS tagging, a python had to have a minimum girth of $24 \mathrm{~cm}$, but we also assessed overall health and body condition before making the decision to GPS-tag a python. Guidelines for animal telemetry usually suggest a device weight should be below a given percentage of the animal's total body weight. The American Society of Ichthyologists and Herpetologists animal care guidelines suggest the maximum weight of a telemetry device should not exceed $10 \%$ of the animal's body weight, and further note that in practice this is often reduced to between $1 \%$ and $6 \%$ [41]. Our minimum girth rule was more than sufficient to ensure that all pythons had a sufficient weight to carry the tags, and in this case, we were more concerned about the relative diameter of the tag to the diameter of the python.

\section{GPS and VHF tags}

We used Quantum 4000E GPS tags manufactured by Telemetry Solutions (Concord, CA). These tags are biologgers, i.e., they store their GPS data onboard and must be physically recovered to download the data. Each tag weighed $50 \mathrm{~g}$, and the tag body had a cylindrical shape, with a length of $6.0 \mathrm{~cm}$ and a diameter of $2.0 \mathrm{~cm}$. The antenna of each tag was $9.5 \mathrm{~cm}$ long. To our knowledge, these are the only GPS biologging tags that are currently available in this small, cylindrical package that lends itself to implantation in a snake.

The GPS tags were initially programmed to attempt GPS fixes (i.e., the tag powered on and attempted to locate GPS satellites and record its position) every $60 \mathrm{~min}$, with each fix attempt lasting up to $90 \mathrm{~s}$. After the first few weeks of the first tracking season, we made the decision to increase the duration of each fix attempt to $120 \mathrm{~s}$ in order to increase the amount of time the tag had to locate the maximum number of satellites and thus improve location precision. In order to maintain expected battery life, we reduced the fix attempt frequency to once every $90 \mathrm{~min}$. We initially programmed the tags for all pythons in the second tracking season with this schedule from the start of that season. Based on manufacturer-provided software estimates, tags were expected to last 3 months with this programming schedule. We accounted for these different schedules when calculating expected fixes throughout this manuscript.

Prior to implantation, the USB port of each GPS tag was closed with a rubber plug and then dipped in a multi-purpose rubber coating for complete waterproofing. Additionally, we tied a small ( $\sim \mathrm{cm}$ diameter) loop of non-absorbable suture to each GPS tag and then rubber dipped the base of the suture to ensure stability. We used this loop of suture during surgery to anchor the tag inside the python's body (see below).

Each python was also implanted with a VHF radio tag to allow us to relocate the animal. We used Holohil model AI-2 transmitters, weighing 25 g each (Holohil Systems, Ltd., Carp, ON, Canada). The combined weight of VHF and GPS tags was therefore $75 \mathrm{~g}$, comfortably under $1 \%$ of all study animals' body weights.

\section{Tag implantation surgeries}

All tag implantation surgeries were performed by Christopher Smith, DVM, in accordance with University of Florida (201508769) and USGS (USGS/SESC 2015-06) Institutional Animal Care and Use Committee (IACUC) protocols.

We anesthetized pythons using isoflurane, an inhalation general anesthetic. Once the animals were fully anesthetized, we began surgery to implant both the GPS tag and a separate VHF radio tag. Both the GPS tag and the VHF radio tag were implanted subcutaneously; however, the GPS tag's antenna needed to exit the body for the tag to function properly (Fig. 1). The tags were both placed in the caudal third of the body, with the incision approximately where the belly scutes transition to body scales. We tightly sutured the skin around the antenna

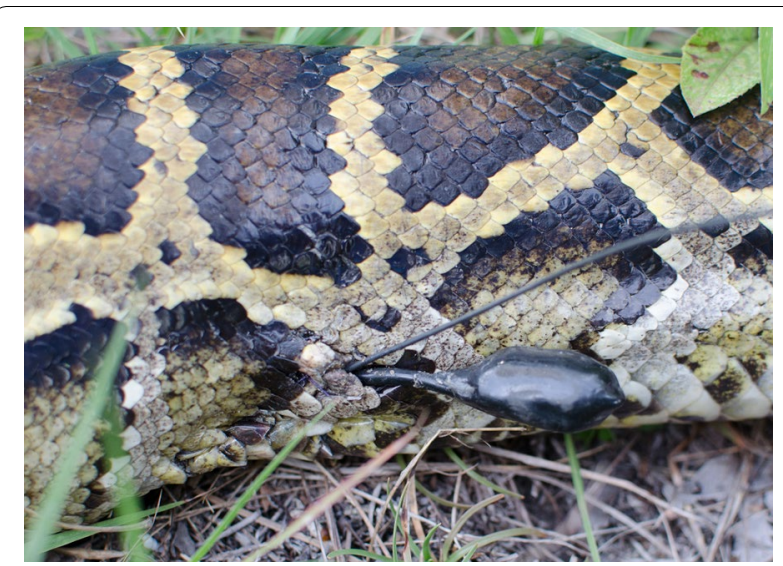

Fig. 1 Photograph of a Burmese python showing the GPS antenna exiting through the skin. GPS signal attenuates under the skin, and therefore, these tags must have an external antenna, potentially compromising the animal's health and tag retention. The large, bulbous antenna is the GPS antenna, and the thin wire is the UHF remote download antenna 
exit site, but this site created a weak point from which the tag could have potentially been expelled. To deal with this issue, we used the loop of non-absorbable suture (described above) to anchor the GPS tag to the muscle surrounding the python's rib cage, thereby reducing the pressure the tag could place on the antenna exit site. The VHF tag was implanted entirely subcutaneously, following standard methods, and was not anchored internally to the rib cage $[18,31,42,43]$. All external surgical openings were closed using both non-absorbable suture and surgical glue to reduce the possibility of any wound opening or infection.

Immediately following the procedure, pythons were placed on a heating pad to recover. Once they regained consciousness and coordination, we transferred them into a clean snake bag and placed them inside a Rubbermaid Action Packer box to recover in a minimal stress environment. We monitored pythons in the laboratory for 24-48 h before we released them back into ENP at their original capture location (Fig. 2).

\section{Tracking seasons}

Pythons were GPS-tracked during both of the two distinct seasons in ENP: the wet season, which lasts roughly from May to October and corresponds biologically to the python non-breeding season, and the dry season, which lasts roughly from November to April and corresponds biologically to the python breeding season [18]. The first tracking season of this study was July 31, 2015-October 31, 2015 (the wet season). The second tracking season was January 1, 2016-April 15, 2016 (the dry season).

Some individual pythons were tracked in both seasons, meaning that the first GPS tag was removed and a tag with a fresh battery was implanted. In those cases, the second tag was implanted on the opposite side of the body to allow the old surgery site to fully heal and eliminate the possibility that old scar tissue would impede the new surgery. The battery on the VHF tags lasted much longer ( 2 years for VHF vs. 3-4 months for GPS), so those tags remained unchanged.

\section{Tracking}

The GPS tag stored location information onboard the tag (i.e., it was a biologging tag) rather than uploading it via satellite, and so the data had to be downloaded either by plugging the tag into a computer via USB port or by using the UHF remote download feature of the tags. The USB option was available only after the tag was removed from the animal after the end of the field season, i.e., USB download of the data required physical recapture of the snake and surgical removal of the tag. For the UHF download option, we needed to be within $2-20 \mathrm{~m}$ of the tagged python, often with a clear line of sight, i.e., data could be recovered from an unrestrained python in situ.

We monitored python locations via VHF radiotelemetry after release to ensure that pythons were moving normally, which based on previous VHF-only tracking meant moving about once a day for the first few days after release, before settling into a less frequent pattern of movement bouts [18]. When we encountered a tagged python in the wild, we attempted to observe the surgery site and external GPS antenna without disturbing the python, and then, we attempted to download the data via UHF. We were able to track and download data from pythons one to two times per week in the first 3-4 weeks of each season, but after that, we lost VHF signal from the road for most study animals, and because of a lack of regular aviation support (see [18, 37] for a discussion of typical aviation support), we could not relocate those missing animals until the end of the tracking season. Flights were in a National Park Service Cessna 206H with permanent amphibious floats. We typically searched for missing animals from altitudes of approximately $2000 \mathrm{ft}$ (around $600 \mathrm{~m}$ ) and then dropped down to approximately $500 \mathrm{ft}$ (around $150 \mathrm{~m}$ ) to pinpoint a location after initially locating the signal. We recaptured pythons as soon as possible after the end of their GPS tracking duration, although some study animals remained missing without regular aviation support. Some healthy, recaptured pythons from the first field season were implanted with new tags and re-released for the second season. Otherwise, all pythons were euthanized at the end of their tracking season.

\section{Quantifying habitat-driven location bias}

Habitat-driven bias in GPS location success is a wellknown issue in GPS tracking [8]. Many habitat features can impact GPS fix success, including dense vegetation, fresh or saltwater, and underground refugia. For this study, we focused on quantifying the effect of vegetation, which we believe to be the most variable factor (i.e., a GPS tag underground or underwater will likely have zero fix success, whereas some functionality is expected even in dense vegetation). To quantify this bias in our tag configuration, we built seven GPS platforms to deploy in situ in ENP under varying levels of vegetation density. Each platform consisted of a small wooden base $(30 \mathrm{~cm} \times 18 \mathrm{~cm} \times 3.8 \mathrm{~cm})$, with a 6.0 -cm-diameter PVC pipe attached on top. A small cutout in the PVC allowed the body of the tag to sit down in the pipe, and then, an ambient temperature freezer gel pack was ducttaped on top of the tag, allowing only the tag's antenna to be exposed, thus simulating implantation in a python (Fig. 3). The tags were programmed with the same fix schedule as those tags implanted in the pythons. 


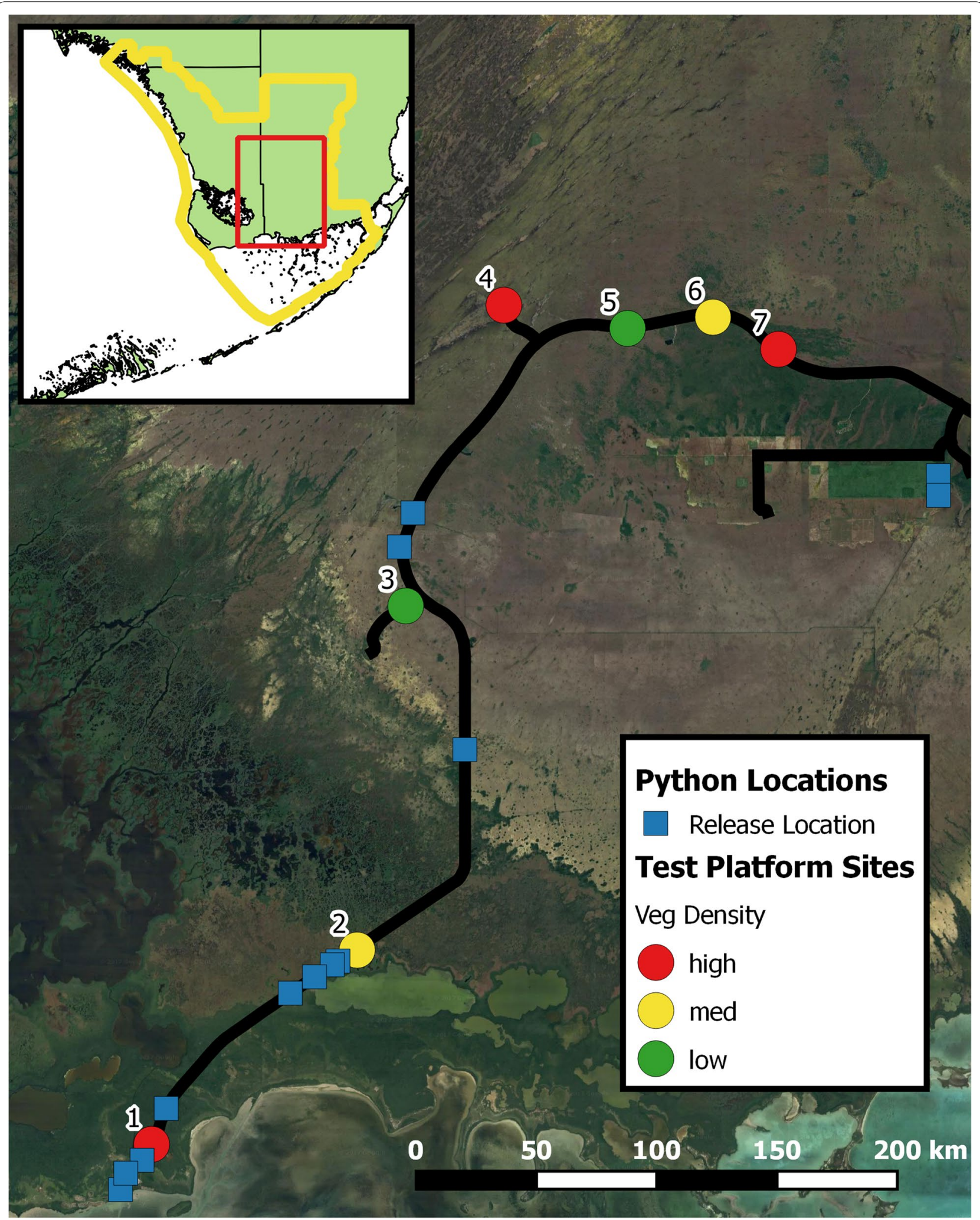

Fig. 2 Map of study area. Map of the study site in Everglades National Park (ENP), FL. GPS-tagged pythons release locations are shown by squares, and GPS test platform sites are shown by colored circles indicating the relative vegetation density at that site. On the inset map, the yellow line is the boundary of ENP and the red line is the extent of the main map panel 


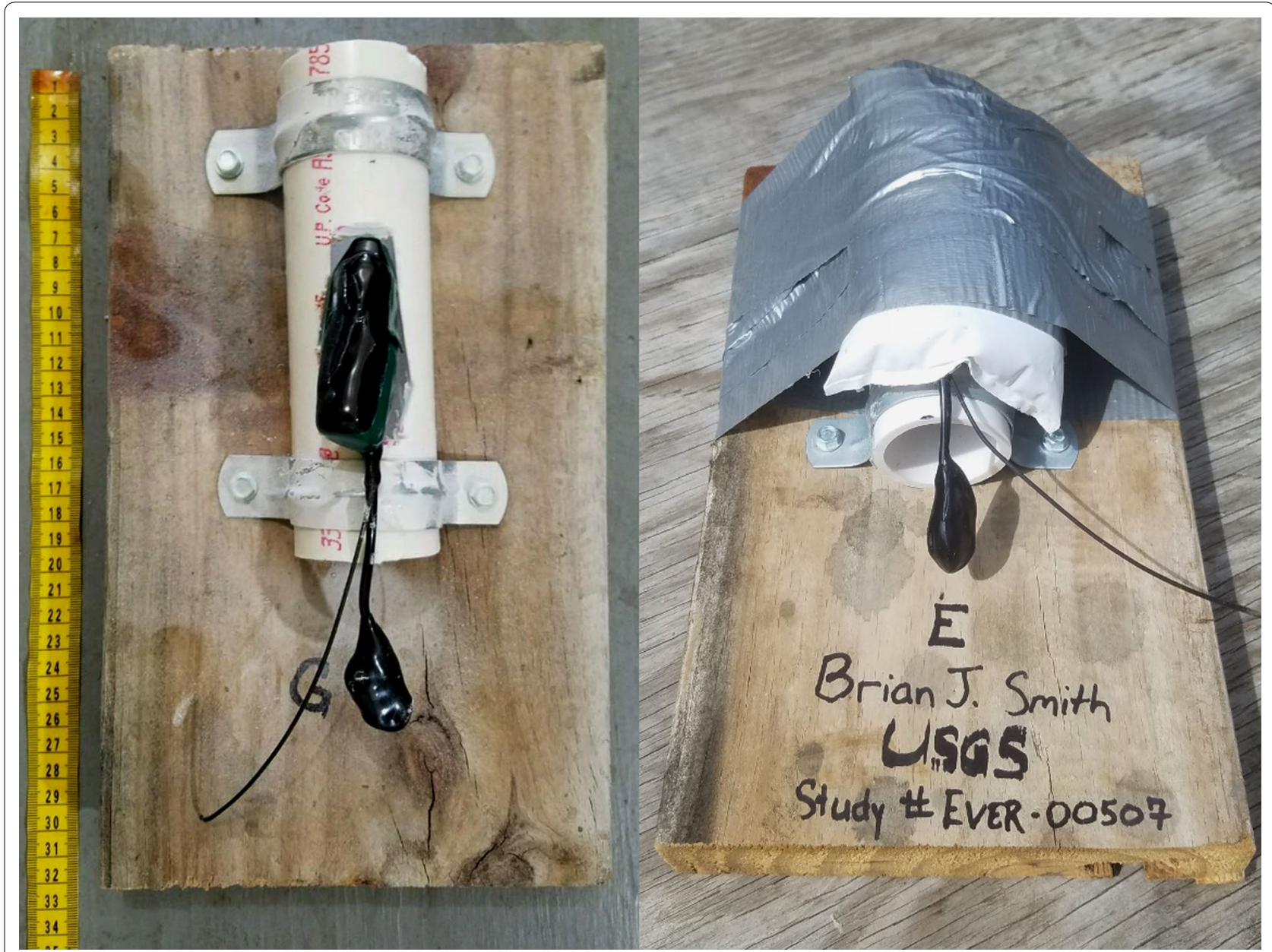

Fig. 3 GPS test platforms. We deployed seven GPS test platforms in ENP to quantify habitat-driven bias in fix rate and measure GPS location accuracy and precision. Tag bodies were mounted inside a cutout in a PVC pipe (left) and then covered with freezer gel packs and duct tape (right) so that only the antenna protruded to simulate implantation under a python's skin

We quantified vegetation density with EVI (enhanced vegetation index) from the MODIS instrument aboard the NASA Terra satellite [44]. The MODIS MOD13Q1 data were retrieved from the online USGS Earth Explorer tool, courtesy of the NASA EOSDIS Land Processes Distributed Active Archive Center (LP DAAC), USGS/Earth Resources Observation and Science (EROS) Center, Sioux Falls, South Dakota (https://earthexplorer.usgs. gov/). The data used to select the tag deployment sites were collected by the satellite between February 19 and March 6, 2017, and downloaded on April 24, 2017. We selected seven sites 50-100 m from the Main Park Road in ENP at which to deploy the test platforms: two sites were designated as low density (EVI $<2600$ ), two sites as medium density (medium $=2600 \leq \mathrm{EVI}<4200$ ), and three sites as high density (EVI $\geq 4200$ ). All tag platforms were placed on dry ground. Although the low-density sites (primarily sawgrass marsh) would have been flooded at the peak of the wet season, it was simply by chance that the areas around MPR were dry at the time of this study. For purposes of data analysis, we downloaded a new EVI dataset collected during the tag deployment, collected by the satellite between May 26 and June 10, 2017, and downloaded on August 7, 2017.

Sites were numbered one through seven, and tags were named A-G (see Fig. 2 for map of study area). Each platform was deployed at each site for 6-8 days, resulting in approximately 112 expected GPS locations per week. After that week, the tags were rotated to the next site, so that at the end of 7 weeks, each tag had been at each location. This allowed us to separate the effect of the vegetation from any possible variability in the individual tags. The week before deployment in ENP, we deployed test platforms at our laboratory in Davie, Florida, with 
a clear view of the sky for a baseline fix rate. The week after collection from ENP, the tags were again deployed at our laboratory with a clear view of the sky to verify that the baseline fix rate had not changed over time (e.g., because of a change in battery power or exposure to the elements). Tag data collection began in Davie on April 26, 2017. Tag platforms were deployed at their first site in ENP on May 3, 2017, and were collected from their final site in ENP on June 22, 2017. Data collection ended on June 28, 2017, after another 6 days in Davie.

\section{Quantifying locational accuracy and precision}

Low positional accuracy and/or precision is a potential problem in GPS tracking [8]. We made use of the habitatbias test platforms to also measure the locational accuracy and precision of the GPS locations. We determined the true location with a Garmin eTrex handheld GPS accurate to $\pm 3 \mathrm{~m}$.

\section{Data analysis}

All analyses and calculations were performed in the open source statistical programming platform $\mathrm{R}$ (version 3.3.2) [45].

\section{Deployment outcomes}

To summarize outcomes for each GPS-tagged python, we recorded the python's final status (i.e., whether or not it had been recovered from the field), the GPS tag's status (i.e., whether we downloaded data from it, whether it malfunctioned and did not record data, or whether the animal lost the tag), the python's release and recovery date, the GPS's scheduled start and end date, the expected number of fixes in that time period, and the actual number of fixes. We then summarized the number of catastrophic failures (i.e., deployments that resulted in no data), the number of successful deployments, and the actual GPS fix rate.

\section{Quantifying habitat-driven location bias}

The habitat-bias test platform data were analyzed using generalized linear mixed models (GLMMs) with tag identity as a random effect to account for any tag variability. All models were fit using the package 'lme4' in $\mathrm{R}$ [46]. We fit two models with binomial fix success for each fix attempt as the response variable: a null model estimating only the average fix rate and a model with EVI as a predictor variable. We checked that the EVI model outperformed the null model using AICc and then used the EVI model to characterize the relationship between vegetation and fix rate. We calculated the marginal $\left(R_{\mathrm{GLMM}(\mathrm{m})}^{2}\right)$ and conditional $\left(R_{\mathrm{GLMM}(\mathrm{c})}^{2}\right)$ pseudo- $R^{2}$ values for the model using the method of Nakagawa and Schielzeth [47]. We used the function 'r.squaredGLMM' from the package 'MuMIn' to do the pseudo- $R^{2}$ calculations [48]. We generated $95 \%$ confidence intervals around the model predictions using the bootstrapping function 'bootMer' in 'lme4' [46].

\section{Quantifying locational accuracy and precision}

We calculated accuracy by first calculating a single mean location from all of the GPS locations for a given tag at a given site, then calculating the distance from that mean location to the true location. We calculated precision as the distance from each single GPS location to the average location of the tag (i.e., the mean tag location from the accuracy measurement). All distance calculations were Euclidean distances calculated on projected points (Universal Transverse Mercator projection, WGS 84 spheroid). We fit both null and EVI linear mixed models to the resulting accuracy and precision data using the package 'ImerTest', which provides p-values for the parameters by basing the degrees of freedom on the Satterthwaite approximation [49]. As with the habitat-bias models, we used tag identity as the random effect, compared the EVI to the null model using AICc, evaluated goodness of fit with pseudo- $R^{2}$, and used bootstrapping to generate confidence intervals.

\section{Results}

\section{Deployment outcomes}

We obtained 10 individual pythons between June 3 and November 17, 2015, that were large enough to accommodate a GPS tag for this study. We released six GPStagged pythons between July 30 and August 1, 2015, for the first tracking season (wet season), and we released seven GPS-tagged pythons-including three individuals (A02, A04, and A06) also tracked in the first season-between November 23 and December 21, 2015, for the second tracking season (dry season; Table 1). Out of 13 total deployments, we were able to recapture ten pythons. The remaining three were never relocated due to lack of aviation support. Five of these ten (50\%) were catastrophic failures; one individual (A02, redeployed for the second season) died before tracking started (cause unknown, but possibly due to the effects of tag surgery), two individuals (A04 in the second season and A08) were recaptured but had expelled their GPS tag, and two individuals (A06 in the second season and A12) had a software malfunction resulting in no data being recorded by the tags. Five pythons-four from the first tracking season and one from the second tracking season-had successful GPS downloads (50\%). One of those five animals, A04 (first season), had apparently damaged its GPS tag on September 23, 2015, over one full month before the expected expiration of the battery (expected October 31 ). The GPS tag continued logging fix attempts, but it never 
Table 1 Summary of each python deployment

\begin{tabular}{|c|c|c|c|c|c|c|c|c|c|}
\hline Python ID & Season & $\begin{array}{l}\text { Python } \\
\text { status }\end{array}$ & GPS status & Release date & $\begin{array}{l}\text { Recovery } \\
\text { date }\end{array}$ & $\begin{array}{l}\text { GPS start } \\
\text { date }\end{array}$ & $\begin{array}{l}\text { GPS end } \\
\text { date }\end{array}$ & $\begin{array}{l}\text { Expected } \\
\text { fixes }\end{array}$ & $\begin{array}{l}\text { Obtained } \\
\text { fixes }\end{array}$ \\
\hline A02 & Wet 2015 & Recovered & Downloaded & $7 / 30 / 2015$ & $11 / 18 / 2015$ & $7 / 30 / 2015$ & $10 / 31 / 2015$ & 1473 & 107 \\
\hline A03 & Wet 2015 & Missing & Unknown & 8/1/2015 & N/A & 8/1/2015 & $10 / 31 / 2015$ & 2010 & N/A \\
\hline A04 & Wet 2015 & Recovered & Downloaded & $7 / 30 / 2015$ & $11 / 24 / 2015$ & $7 / 30 / 2015$ & $9 / 23 / 2015^{c}$ & $866^{c}$ & 70 \\
\hline A05 & Wet 2015 & Missing & Unknown & $8 / 1 / 2015$ & $\mathrm{~N} / \mathrm{A}$ & $8 / 1 / 2015$ & $10 / 31 / 2015$ & 1448 & N/A \\
\hline A06 & Wet 2015 & Recovered & Downloaded & $7 / 30 / 2015$ & $12 / 1 / 2015$ & $7 / 30 / 2015$ & $10 / 31 / 2015$ & 1452 & 463 \\
\hline A07 & Wet 2015 & Recovered & Downloaded & $8 / 1 / 2015$ & $11 / 24 / 2015$ & $8 / 1 / 2015$ & $10 / 31 / 2015$ & 1447 & 266 \\
\hline $\mathrm{AO}^{\mathrm{a}}$ & Dry 2016 & Died in field & No data ${ }^{b}$ & $11 / 23 / 2015$ & $1 / 20 / 2016$ & 1/1/2016 & $3 / 31 / 2016$ & 1456 & 0 \\
\hline $\mathrm{A} 04^{\mathrm{a}}$ & Dry 2016 & Recovered & Expelled tag ${ }^{b}$ & $12 / 21 / 2015$ & $5 / 18 / 2016$ & $1 / 1 / 2016$ & $3 / 31 / 2016$ & 1456 & 0 \\
\hline $\mathrm{A} 06^{\mathrm{a}}$ & Dry 2016 & Recovered & $\begin{array}{l}\text { Malfunc- } \\
\text { tioned }^{b}\end{array}$ & $12 / 21 / 2015$ & $4 / 1 / 2016$ & $1 / 1 / 2016$ & $3 / 31 / 2016$ & 1456 & 0 \\
\hline A08 & Dry 2016 & Recovered & Expelled tag ${ }^{b}$ & $11 / 23 / 2015$ & $9 / 14 / 2016$ & $1 / 1 / 2016$ & $3 / 31 / 2016$ & 1456 & 0 \\
\hline A09 & Dry 2016 & Recovered & Downloaded & $11 / 23 / 2015$ & $9 / 14 / 2016$ & $1 / 1 / 2016$ & $3 / 31 / 2016$ & 1456 & 363 \\
\hline A11 & Dry 2016 & Missing & Unknown & $12 / 21 / 2015$ & $\mathrm{~N} / \mathrm{A}$ & $1 / 1 / 2016$ & $3 / 31 / 2016$ & 1456 & N/A \\
\hline A12 & Dry 2016 & Recovered & $\begin{array}{l}\text { Malfunc- } \\
\text { tioned }^{b}\end{array}$ & $12 / 21 / 2015$ & $2 / 24 / 2017$ & $1 / 1 / 2016$ & $3 / 31 / 2016$ & 1456 & 0 \\
\hline
\end{tabular}

a Individual python redeployed for a second season

b Outcome considered catastrophic failure

c Adjusted down for tag malfunction after September 23, 2015

successfully obtained another fix in the following month. Therefore, six out of ten deployments resulted in some sort of failure, and four out of ten deployments resulted in a full dataset as expected.

Of the five pythons whose tags yielded a dataset, the average expected number of GPS fixes (adjusting A04's end date to September 23) was 1338.8 (range 866-1473), and the average actual number of GPS fixes was 253.8 (range 70-463; Fig. 4). Therefore, fix rate was generally low, averaging $18.1 \%$ (range $7.2-31.8 \%$ ). Average location frequency was 14.5 locations/animal/week (range $4.0-26.1)$.

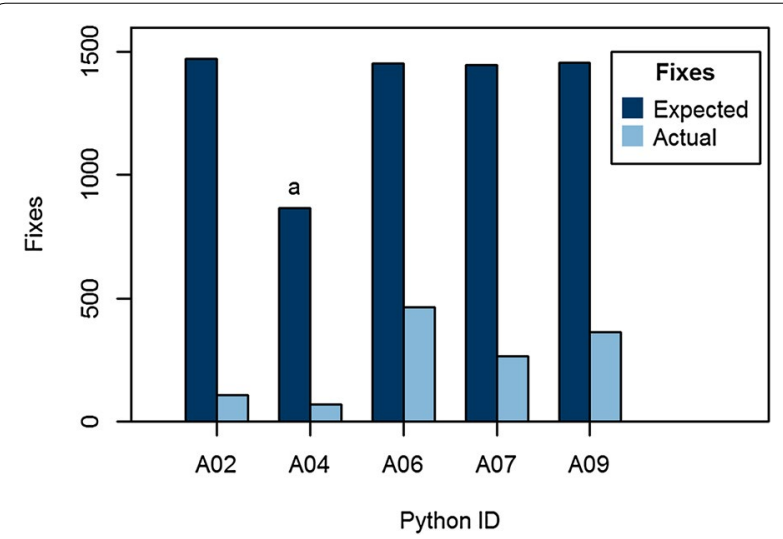

Fig. 4 GPS expected and actual fixes. Expected number of GPS fixes based on tag programming schedule and actual number of successful fixes obtained by the python GPS tags. ${ }^{a}$ Adjusted end date because of tag malfunction on September 25, 2015

\section{Quantifying habitat-driven location bias}

The EVI model clearly outperformed the null model $(\triangle \mathrm{AICc}=100.77)$. The model explained a moderate proportion of the variance observed, with $R_{\mathrm{GLMM}(\mathrm{m})}^{2}=0.235$ and $R_{\mathrm{GLMM}(\mathrm{c})}^{2}=0.463$. The model showed that fix success was near $100 \%$ for EVI $<4000$, declining to a fix success near $0 \%$ for EVI approaching the maximum value of 10,000 (Fig. 5). The theoretical range of EVI values are from - 2000 to 10,000, but the range of non-water values observed in our dataset within ENP was 1-9622 (mean $=2467, \mathrm{SD}=1378)$.

\section{Quantifying locational accuracy and precision}

Overall mean accuracy was $7.3 \mathrm{~m}$ (95\% CI 3.4-11.2 m, $n=54)$. Accuracy values ranged from 0.0 to $95.0 \mathrm{~m}$, but $97.5 \%$ of values were $\leq 35.9 \mathrm{~m}$. Overall mean precision was $12.9 \mathrm{~m}$ (95\% CI $8.7-17.1 \mathrm{~m}, n=5830)$. Precision values ranged from 0.0 to $6401.4 \mathrm{~m}$, but $97.5 \%$ of precision values were $\leq 37.0 \mathrm{~m}$. Visual inspection of accuracy and precision showed that both decreased with vegetation density (i.e., the values became larger; Fig. 6). The EVI model for accuracy slightly outperformed the null model $(\triangle \mathrm{AICc}=2.51)$, but the $p$ value for the slope parameter was large $(p=0.38)$, so the relationship was not statistically significant. The EVI model for precision performed better against its respective null model $(\triangle \mathrm{AICc}=12.83)$, and the estimated slope had a small $p$ value (slope $=8.98, p<0.001$ ), but the overall fit was not very good with $R_{\mathrm{GLMM}(\mathrm{m})}^{2}=0.002$ and $R_{\mathrm{GLMM}(\mathrm{c})}^{2}=0.012$. 


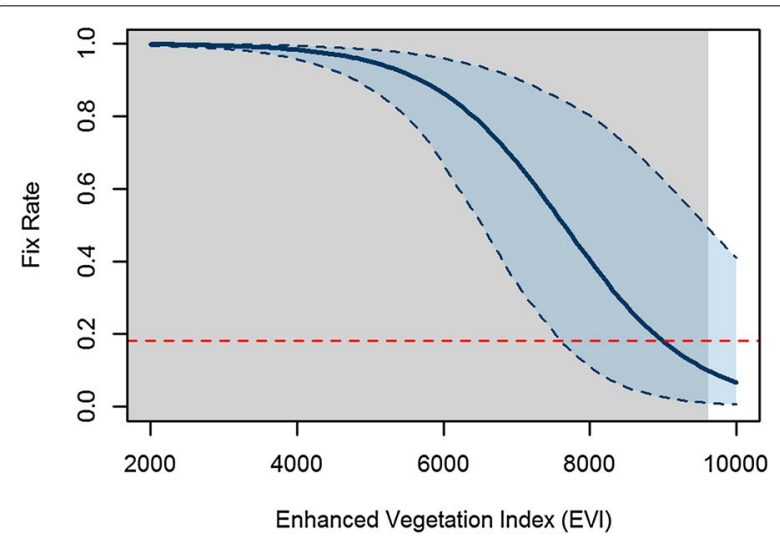

Fig. 5 GPS fix rate versus vegetation density. Model predictions from the GLMM relating fix rate to enhanced vegetation index (EVI), and bootstrapped 95\% confidence envelope. Fix rate declines beginning at $E V I s \approx 4000$ falls to near $0 \%$ at the maximum EVI of 10,000; however, the range of values actually observed in Everglades National Park was from 1 to 9622 (light gray shading). The observed mean fix rate for all pythons deployed was 0.181 (red dashed line). We attribute the discrepancy between predicted and observed fix rate to microhabitat selection by pythons, i.e., pythons spending time underground or underwater and thus exhibiting lower fix rates than predicted by vegetation alone

\section{Discussion}

\section{GPS advantages}

GPS has several advantages over VHF radio tracking. GPS tracking in this study resulted in an average of 14.5 locations/animal/week, a marked improvement over the VHF-only one location/animal/week reported in the past $[18,37]$. This improved location frequency will allow us to estimate differences in resource utilization throughout the day and at a finer behavioral scale. GPS and VHF tracking are also not mutually exclusive: supplementing highly accurate and unbiased visual locations (obtained via homing to the VHF signal) with frequent GPS locations can add valuable information to our understanding of Burmese python spatial ecology, and the resulting dataset could be used to reveal fine-scale behavior by pythons, such as habitat selection by pythons along their movement paths $[50,51]$. There is strong potential in this approach if we associate these steps with not just habitat variables, but also meteorological variables like temperature or barometric pressure, temporal variables like season of the year or time of the day, and variables describing the internal state of the python, such as body temperature or time since last meal. This could also improve our ability to predict python movements and thus optimize our removal efforts.

Accuracy and precision are generally very good for the tags, with mean accuracy $=7.3 \mathrm{~m}$ and mean precision $=12.9 \mathrm{~m}$. These values are sufficient to be useful for understanding python spatial ecology at a novel spatial and temporal scale. This accuracy and precision are much better than what could be achieved with VHF triangulation or aerial telemetry, although not as good as could be achieved with direct observation (e.g., visual location after homing to the VHF signal, as described in [37]). Accuracy and precision seemed to decrease with vegetation density (Fig. 6), although the model we used to describe the relationship is noisy at best and may require further examination.

\section{GPS drawbacks \\ Catastrophic failures}

GPS tracking of Burmese pythons also has some drawbacks. Half of our deployments resulted in catastrophic failures, an unfortunate phenomenon that many studies report [8]. Without frequent VHF tracking and additional sensors, we know almost nothing about the paths of these five animals, further reducing sample sizes and the ability to generalize findings to the population level. We believe that our catastrophic failure rate is unacceptably high, and we suggest some steps that should be taken to reduce this rate in future studies.

First, the relatively bulky tag (compared to the VHF tag) and the external antenna put extra strain on the python. One of our study animals (A02) died in the field shortly after her second deployment. While we could not determine the exact cause of death, it is possible that the second surgery could have been a contributing factor. None of the other catastrophic failures were related to the study animal's health, but nevertheless, we do not recommend putting the same animal through multiple surgeries in consecutive seasons in the future. Two other animals (A04-dry season deployment, A08) were able to tear the tag out of their bodies, likely by catching the bulbous GPS antenna (see Fig. 1) on some vegetation or substrate and then continuing to move forward. This demonstrates that the antenna hole is a critical weak point in tag attachment, a problem that needs to be solved before future GPS applications in large constrictors. We suggest reconfiguring the GPS antenna to eliminate the bulbous end, and we suggest applying extra non-absorbable suture at the antenna exit point. Overall, the surgery sites looked to be in good condition (with the notable exception of one of the two animals that expelled their tag), with minimal irritation of the internal tissue around the GPS tag. Future research should examine the health of animals with an external antenna, the resulting microbiome inside the tag capsule, and find ways to mitigate any adverse effects.

An additional two catastrophic failures occurred because of tag malfunction. While we cannot determine the exact cause of these failures, we can suggest 


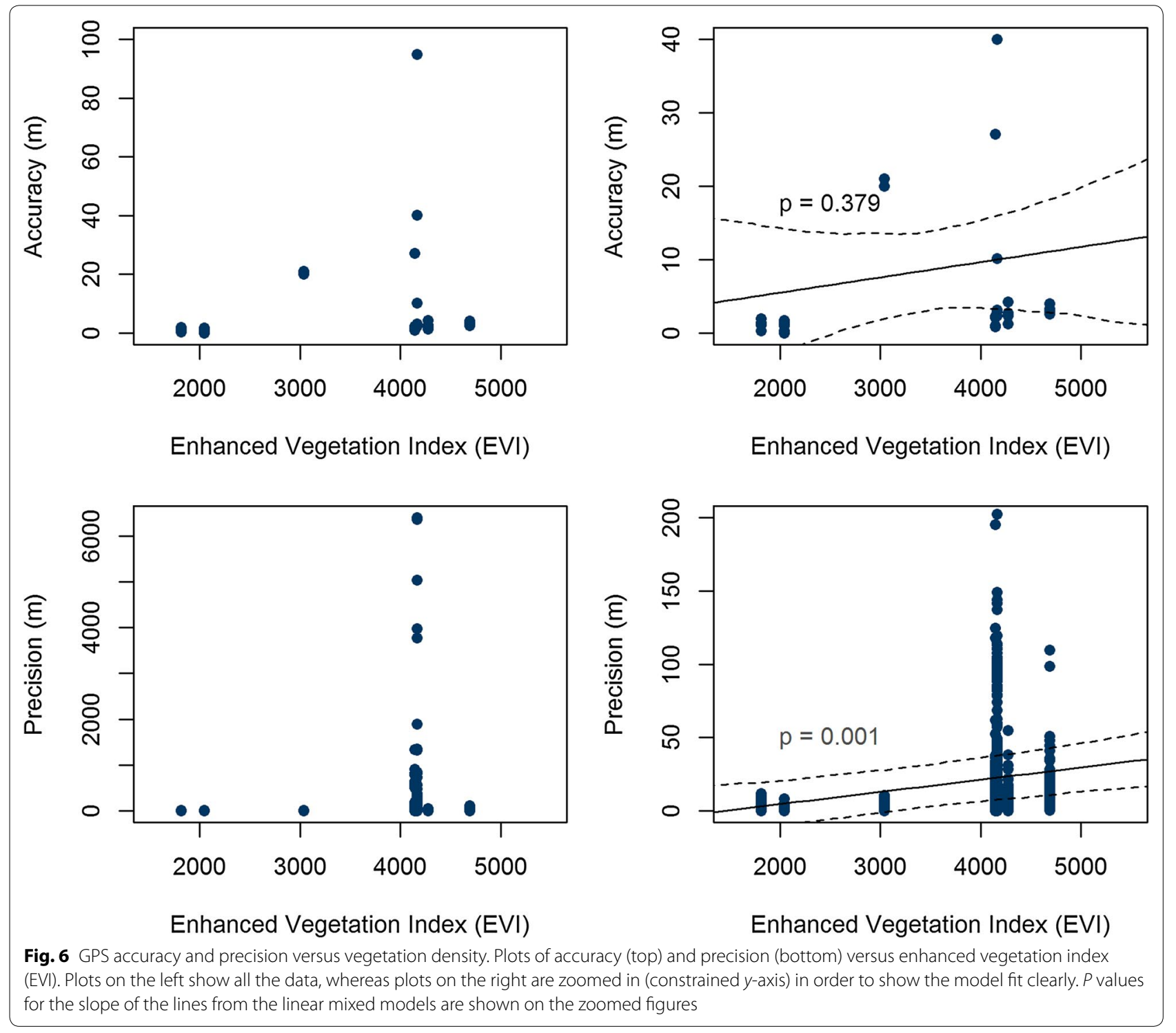

additional weaknesses that should be addressed. Our experience in field testing these tags suggests that the solder point where the antenna attaches to the body of the tag is weak. Antenna connectivity is crucial in these tags, so strengthening that juncture should be a priority. Again, eliminating the bulbous end of the antenna will help prevent that from being caught on vegetation and will contribute to alleviating this issue as well. Additionally, the software and firmware for configuring the GPS hardware has some peculiarities. For example, a failure of the user to set the tag's internal clock will result in a rapid series of attempts to contact the GPS satellites in order to set the clock. In the event that the tag cannot get reception, it will quickly drain $100 \%$ of its battery searching. Future tag models should have firmware that reduces the chance a small human error can render the tag useless.

\section{Habitat effects}

Habitat-driven bias in GPS fixes can cause problems with interpreting movement paths or estimating resource selection, but other measures, like home ranges, are more robust to this error [8]. Methods exist to cope with this type of bias, such as sample weighting, simulation, or pairing GPS tracking with regular VHF tracking $[7,52]$. Recio et al. [11] showed that the effects of vegetation, topography, and animal behavior on fix rates is speciesand system-specific, so research into understanding the fix rates under different environmental conditions is needed for large constrictors. Future implementations of GPS tracking in large constrictors should be paired with VHF homing and visual location to complement the GPS track and provide unbiased data in relation to habitat and inform microhabitat selection. As we have already 
suggested, more locations per week would help quantify habitat bias (we suggest three to four locations per week would be a good compromise between logistics and estimation). Further complementing the track with other biologging sensors, such as accelerometers or network nodes, could increase the resolution of tracking and help researchers and managers understand the fine details of when and where pythons move.

Observed fix rates in python tags were lower than what we would predict based on vegetation density alone, with the observed fix rate of $18.1 \%$ occurring only in the densest vegetation in ENP (Fig. 5). We attribute this discrepancy to microhabitat selection by pythons. Intensive VHF tracking with visual observations has shown that pythons spend a significant amount of time underground or underwater. This would greatly hamper GPS performance and lead to the observed low fix rate. At the time of our test platform deployments, there were no raster cells in ENP that had EVI > 9622, indicating that at even at the most densely vegetated places in the study area, python tags should have at least some $(\sim 10 \%)$ fix success. The overall low fix success is likely attributable to a mixture of macrohabitat and microhabitat effects and cannot be determined without additional (microhabitat) information.

Under conditions of dense vegetation, GPS accuracy and precision can drop dramatically. We observed precision values poorer than $1.0 \mathrm{~km}$ on a very small subset of locations ( 9 out of 5830 , or $0.2 \%$ of the observations). All of our accuracy measurements were $<100 \mathrm{~m}$, which we consider very good, but single erroneous points (imprecise locations) still need to be removed from the dataset before analyzing animal trajectories.

\section{Comparison to automated telemetry}

Ward et al. [53] evaluated the use of automated VHF telemetry to track the movements of ratsnakes (Pantherophis spp.) using multiple directional antennas on towers. They were able to use the automated system to attempt to record a location for each snake every 3-5 min, a much higher frequency than we programmed our GPS tags with. After filtering their data, they were left with $30.6 \%$ of the records available for analysis, roughly analogous to a fix rate, although we expect to still filter a small percentage of our GPS fixes for imprecision. Their reported 'fix rate' of $30.6 \%$ is much better than our fix rate of $18.1 \%$. They also evaluated the accuracy of the system by placing stationary test tags in the core area nearest their towers, in the secondary area farther from their towers, and outside the secondary area. They found average accuracy in these areas to be $28.6,77.1$, and $142.9 \mathrm{~m}$, respectively. This is much worse than our mean accuracy of $7.3 \mathrm{~m}$, and furthermore, GPS tags are not constrained by proximity to a tower. The authors also cite the high cost of the automated system (approximately $\$ 7300$ US per antenna/ recording unit combination) as a drawback. For comparison, each of our GPS tags costs approximately \$2000 US. Pythons also have large home ranges in the Everglades (2250 ha, see [18]), and the maximum range for VHF reception from approximately ground level is $<0.5 \mathrm{~km}$ (BJS, personal observation), making it cost-prohibitive to try to track these large snakes using stationary towers. Overall, automated telemetry does exhibit higher fix rates, but also has less accuracy and higher cost than GPS telemetry.

\section{Future directions}

Filtering erroneous or imprecise locations remains a major challenge in working with GPS telemetry data [8]. Common approaches to filtering include biological filters based on a maximum speed or filters based on sharp turning angles [8]. Manual inspection of a dataset is also possible, but this is time-consuming with large GPS datasets. Because of the inherently subjective nature of this process, automated filtering routines should be considered first. Increased frequency of VHF locations would help to identify true locations and calibrate filtering algorithms.

\section{Conclusions}

Our GPS tracking of Burmese python did provide more locations/animal/week than previous VHF radio tracking could provide, and the accuracy and precision of the tags were generally very good. Therefore, we concluded that GPS biologging technology can provide the data necessary to answer new questions about large constrictor spatial ecology.

As invasive Burmese pythons spread north into the Greater Everglades Ecosystem and beyond, the availability of very different habitat types could completely alter the way this technology performs. GPS fix success might be better in upland pine habitats with a sparse canopy and less standing water, or it might be worse if pythons have increased access to underground refugia. Further investigation into both python spatial ecology and GPS performance throughout their invaded range is important for developing an understanding of threats to our native communities and management options.

More generally, large constrictors occupy a diverse range of habitat types throughout the world-from jungles and wetlands to arid scrub-and understanding the performance of tracking technology in each study site will be crucial to using that technology to answer important ecological questions. GPS tracking has the potential to be another tool for researchers to improve our understanding of large constrictor spatial ecology, but it should 
not be thought of as a panacea on its own. With complementary tracking data, applications in different habitat types, and careful attention to data filtering, GPS technology can help us build a more complete picture of how large constrictors use space in their environment, providing insight into their biotic requirements and internal states.

As GPS and battery technologies are further miniaturized, GPS tracking will become a possibility for more taxa that are cryptic and select challenging microhabitats like large constrictors. In those cases, the performance of the technology should be comprehensively evaluated before data from those tags are used to draw biological inferences.

\section{Authors' contributions}

All coauthors contributed to writing and editing the manuscript. BJS designed the procedures, performed field work, analyzed the data, and created the figures. $\mathrm{KMH}$ conceived the study, provided input on study design, and secured funding. FJM provided input on study design. MB provided input on study design and data analysis. CMR supervised the research development and provided input on study design. All authors read and approved final manuscript.

\section{Author details \\ ${ }^{1}$ Cherokee Nation Technologies, Davie, FL, USA. ${ }^{2}$ Wetland and Aquatic Research Center, US Geological Survey, Davie, FL, USA. ${ }^{3}$ Department of Wild- life Ecology and Conservation, Fort Lauderdale Research and Education Center, University of Florida, Davie, FL, USA. ${ }^{4}$ Department of Wildlife Ecology and Conservation, University of Florida, Gainesville, FL, USA.}

\section{Acknowledgements}

We thank Everglades National Park, especially Tylan Dean, for help with permitting and logistical support. Special thanks to USGS Invasive Species Branch employees stationed in ENP for helping us obtain study animals. Numerous volunteers and researchers helped by catching study animals, particularly Tom Rahill and the Swamp Apes, as well as Melissa Miller and Mario Aldecoa. Thomas Selby, Devon Nemire-Pepe, David Roche, Mike Cherkiss, and Andrew Crowder provided field help. Drs. Christopher Smith and Andrea Zimandy performed tag implantation surgeries and provided valuable animal care support and guidance throughout. Bryan Falk provided a valuable review of this manuscript. We thank editor Brian Todd and two anonymous reviewers for their time and thoughtful comments. Any use of trade, product, or firm names is for descriptive purposes only and does not imply endorsement by the US Government.

\section{Competing interests}

The authors declare that they have no competing interests.

\section{Availability of data and materials}

The datasets generated and analyzed during the current study are available in the USGS ScienceBase repository, https://doi.org/10.5066/f7h41 qb6.

\section{Consent for publication}

Not applicable.

\section{Ethics approval and consent to participate}

All research was approved by University of Florida (201508769) and USGS (USGS/SESC 2015-06) Institutional Animal Care and Use Committees (IACUCs).

\section{Funding}

This project was funded in significant part by USGS's Greater Everglades Priority Ecosystems Science (GEPES) Program. Additional funding was provided by the USGS Invasives Program. The funding sources have had no role in study design, data collection, data analysis, interpretation of data, or writing the manuscript.

\section{Publisher's Note}

Springer Nature remains neutral with regard to jurisdictional claims in published maps and institutional affiliations.

Received: 28 August 2017 Accepted: 29 January 2018

Published online: 13 February 2018

\section{References}

1. Cooke SJ, Hinch SG, Wikelski M, Andrews RD, Kuchel L, Wolcott TG, et al. Biotelemetry: a mechanistic approach to ecology. Trends Ecol Evol. 2004;19:334-43. https://doi.org/10.1016/j.tree.2004.04.003.

2. Rutz C, Hays GC. New frontiers in biologging science. Biol Lett. 2009;5:289-92. https://doi.org/10.1098/rsbl.2009.0089.

3. Klimley AP. Why publish animal biotelemetry? Anim Biotelemetry. 2013;1:2-4.

4. Martin J, Tolon V, Van Moorter B, Basille M, Calenge C. On the use of telemetry in habitat selection studies. In: Barculo D, Daniels J, editors. Telemetry: research, technology and applications. Hauppauge, NY: Nova Science Publishers, Inc: 2009. p. 37-55.

5. Tomkiewicz SM, Fuller MR, Kie JG, Bates KK. Global positioning system and associated technologies in animal behaviour and ecological research. Philos Trans R Soc B Biol Sci. 2010;365:2163-76. https://doi. org/10.1098/rstb.2010.0090.

6. Onorato DP, Criffield M, Lotz M, Cunningham M, McBride R, Leone $\mathrm{EH}$, et al. Habitat selection by critically endangered Florida panthers across the diel period: implications for land management and conservation. Anim Conserv. 2011;14:196-205. https://doi. org/10.1111/j.1469-1795.2010.00415.x

7. Hebblewhite M, Haydon DT. Distinguishing technology from biology: a critical review of the use of GPS telemetry data in ecology. Philos Trans R Soc B Biol Sci. 2010;365:2303-12. https://doi.org/10.1098/ rstb.2010.0087.

8. Frair JL, Fieberg J, Hebblewhite M, Cagnacci F, DeCesare NJ, Pedrotti L. Resolving issues of imprecise and habitat-biased locations in ecological analyses using GPS telemetry data. Philos Trans R Soc B Biol Sci. 2010;365:2187-200. https://doi.org/10.1098/rstb.2010.0084.

9. Cagnacci F, Boitani L, Powell RA, Boyce MS. Animal ecology meets GPSbased radiotelemetry: a perfect storm of opportunities and challenges. Philos Trans R Soc B Biol Sci. 2010;365:2157-62. https://doi.org/10.1098/ rstb.2010.0107.

10. Latham ADM, Latham MC, Anderson DP, Cruz J, Herries D, Hebblewhite M. The GPS craze: six questions to address before deciding to deploy GPS technology on wildlife. N Z J Ecol. 2015;39:143-53.

11. Recio MR, Mathieu R, Denys P, Sirguey P, Seddon PJ. Lightweight GPS-tags, one giant leap for wildlife tracking? An assessment approach. PLOS ONE. 2011;6:e28225. https://doi.org/10.1371/journal.pone.0028225.

12. Schofield G, Bishop CM, MacLean G, Brown P, Baker M, Katselidis KA, et al. Novel GPS tracking of sea turtles as a tool for conservation management. J Exp Mar Bio Ecol. 2007;347:58-68. https://doi.org/10.1016/j. jembe.2007.03.009.

13. Rosenblatt AE, Heithaus MR, Mazzotti FJ, Cherkiss M, Jeffery BM. Intra-population variation in activity ranges, diel patterns, movement rates, and habitat use of American alligators in a subtropical estuary. Estuar Coast Shelf Sci. 2013;135:182-90. https://doi.org/10.1016/j. ecss.2013.10.008.

14. Campbell HA, Dwyer RG, Irwin TR, Franklin CE. Home range utilisation and long-range movement of estuarine crocodiles during the breeding and nesting season. PLoS ONE. 2013;8:e62127. https://doi.org/10.1371/ journal.pone.0062127.

15. Dwyer RG, Campbell HA, Irwin TR, Franklin CE. Does the telemetry technology matter? Comparing estimates of aquatic animal space-use generated from GPS-based and passive acoustic tracking. Mar Freshw Res. 2015:66:654-64.

16. Price-Rees SJ, Brown GP, Shine R. Spatial ecology of bluetongue lizards (Tiliqua spp.) in the Australian wet-dry tropics. Austral Ecol. 2013;38:493503. https://doi.org/10.1111/j.1442-9993.2012.02439.x. 
17. Flesch JS, Duncan MG, Pascoe JH, Mulley RC. A simple method of attaching GPS tracking devices to free-ranging lace monitors (Varanus varius). Herpetol Conserv Biol. 2009;4:411-4.

18. Hart KM, Cherkiss MS, Smith BJ, Mazzotti FJ, Fujisaki I, Snow RW, et al. Home range, habitat use, and movement patterns of non-native Burmese pythons in Everglades National Park, Florida, USA. Anim Biotelemetry. 2015;3:8.

19. Henderson RW, Powell R. Biology of the boas and pythons. Eagle Mountain: Eagle Mountain Publishing; 2007.

20. Pearson D, Shine R, Williams A. Spatial ecology of a threatened python (Morelia spilota imbricata) and the effects of anthropogenic habitat change. Austral Ecol. 2005;30:261-74.

21. Heard GW, Black D, Robertson P. Habitat use by the inland carpet python (Morelia spilota metcalfei: Pythonidae): Seasonal relationships with habitat structure and prey distribution in a rural landscape. Austral Ecol. 2004;29:446-60.

22. Luiselli L, Bonnet X, Rocco M, Amori G. Conservation implications of rapid shifts in the trade of wild African and Asian pythons. Biotropica. 2012;44:569-73. https://doi.org/10.1111/j.1744-7429.2011.00842.x.

23. Snow RW, Krysko KL, Enge KM, Oberhofer L, Warren-Bradley A, Wilkins L. Introduced populations of Boa constrictor (Boidae) and Python molurus bivittatus (Pythonidae) in southern Florida. In: Henderson RW, Powell R, editors. Biology of boas and pythons. Eagle Mountain: Eagle Mountain Publishing; 2007. p. 365-86.

24. Slip DJ, Shine R. Habitat use, movements and activity patterns of freeranging diamond pythons, Morelia spilota spilota (Serpentes, Boidae): a radiotelemetric study. Aust Wildl Res. 1988;15:515-31.

25. Shine R, Fitzgerald M. Large snakes in a mosaic rural landscape: the ecology of carpet pythons Morelia spilota (Serpentes: Pythonidae) in coastal eastern Australia. Biol Conserv. 1996;76:113-22.

26. Pearson D, Shine R, Williams A. Thermal biology of large snakes in coo climates: a radio-telemetric study of carpet pythons (Morelia spilota imbricata) in south-western Australia. J Therm Biol. 2003;28:117-31.

27. Rivas JA, Molina CR, Corey SJ, Burghardt GM. Natural history of neonatal green anacondas (Eunectes murinus): a chip off the old block. Copeia. 2016;104:402-10. https://doi.org/10.1643/CE-15-238.

28. Alexander GJ, Maritz B. Sampling interval affects the estimation of movement parameters in four species of African snakes. J Zool. 2015;297:30918. https://doi.org/10.1111/jzo.12280.

29. Chiaraviglio M. The effects of reproductive condition on thermoregulation in the Argentina boa constrictor (Boa constrictor occidentalis) (Boidae). Herpetol Monogr. 2006;20:172. https://doi. org/10.1655/0733-1347(2007)20[172:teorco]2.0.co;2.

30. Rahman SC, Jenkins CL, Trageser SJ, Rashid SMA. Radio-telemetry study of Burmese python (Python molurus bivittatus) and elongated tortoise (Indotestudo elongata) in Lawachara National Park, Bangladesh: a preliminary observation. In: Khan MAR, Ali MS, Feeroz MM, Naser MN, editors. The Festschrift of the 50th Anniversary of the IUCN Red List of Threatened Species. Dhaka, Bangladesh: UCN, International Union for Conservation of Nature; 2014. p. 54-62.

31. Reinert HK, Cundall D. An improved surgical implantation method for radio-tracking snakes. Copeia. 1982;1982:702-5. https://doi. org/10.2307/1444674

32. Walters TM, Mazzotti FJ, Fitz HC. Habitat selection by the invasive species burmese python in Southern Florida. J Herpetol. 2016;50:50-6. https:// doi.org/10.1670/14-098.

33. Bruton MJ, McAlpine CA, Smith AG, Franklin CE. The importance of underground shelter resources for reptiles in dryland landscapes: a woma python case study. Austral Ecol. 2014;39:819-29. https://doi.org/10.1111/ aec.12150.

34. Hart KM, Schofield PJ, Gregoire DR. Experimentally derived salinity tolerance of hatchling Burmese pythons (Python molurus bivittatus) from the Everglades, Florida (USA). J Exp Mar Bio Ecol. 2012;413:56-9.
35. Willson JD, Dorcas ME, Snow RW. Identifying plausible scenarios for the establishment of invasive Burmese pythons (Python molurus) in Southern Florida. Biol Invasions. 2011;13:1493-504.

36. Dorcas ME, Willson JD. Hidden giants: problems associated with studying secretive invasive pythons. In: Lutterschmidt WI, editor. Reptiles in research: investigations of ecology, physiology, and behavior from desert to sea. Hauppage: Nova Science Publishers, Inc.; 2013.

37. Smith BJ, Cherkiss MS, Hart KM, Rochford MR, Selby TH, Snow RW, et al. Betrayal: radio-tagged Burmese pythons reveal locations of conspecifics in Everglades National Park. Biol Invasions. 2016;18:3239-50. https://doi. org/10.1007/s10530-016-1211-5.

38. Dorcas ME, Willson JD, Reed RN, Snow RW, Rochford MR, Miller MA, et al. Severe mammal declines coincide with proliferation of invasive Burmese pythons in Everglades National Park. Proc Natl Acad Sci USA. 2012;109:2418-22. https://doi.org/10.1073/pnas.1115226109.

39. McCleery RA, Sovie A, Reed RN, Cunningham MW, Hunter ME, Hart KM. Marsh rabbit mortalities tie pythons to the precipitous decline of mammals in the Everglades. Proc R Soc B Biol Sci. 2015;282:20150120. https:// doi.org/10.1098/rspb.2015.0120.

40. Sovie AR, McCleery RA, Fletcher RJ, Hart KM. Invasive pythons, not anthropogenic stressors, explain the distribution of a keystone species. Biol Invasions. 2016;18:3309-18. https://doi.org/10.1007/s10530-016-1221-3.

41. Beaupre SJ, Jacobson ER, Lillywhite HB, Zamudio K. Guidelines for the use of live amphibians and reptiles in field and laboratory research. Miami: Herpetological Animal Care and Use Committee, American Society of Ichthyologists and Herpetologists; 2004.

42. Hardy DL Sr, Greene HW. Surgery on rattlesnakes in the field for implantation of transmitters. Son Herpetol. 1999;12:25-7.

43. Anderson CD, Talcott M. Clinical practice versus field surgery: a discussion of the regulations and logistics of implanting radiotransmitters in snakes. Wildl Soc Bull. 2006;34:1470-1. https://doi. org/10.2193/0091-7648(2006)34[1470:cpvfsa]2.0.co;2.

44. Didan K. MOD13Q1 MODIS/Terra Vegetation Indices 16-Day L3 Global 250 m SIN Grid V006. 2015.

45. R Core Team. R: a language and environment for statistical computing. R: a language and environment for statistical computing. 2016. http:// www.r-project.org.

46. Bates D, Mächler M, Bolker B, Walker S. Fitting linear mixed-effects models using Ime4. J Stat Softw. 2014;67:51. https://doi.org/10.18637/jss.v067.i01.

47. Nakagawa S, Schielzeth H. A general and simple method for obtaining $\mathrm{R}^{2}$ from generalized linear mixed-effects models. Methods Ecol Evol. 2013:4:133-42

48. Barton K. MuMIn: Multi-model inference. 2016. https://cran.r-project.org/ package $=$ MuMIn.

49. Kuznetsova A, Bruun Brockhoff P, Haubo Bojesen christensen R. ImerTest: tests in linear mixed effects models. 2016. https://cran.r-project.org/ package $=$ ImerTest.

50. Thurfell H, Ciuti S, Boyce MS. Applications of step-selection functions in ecology and conservation. Mov Ecol. 2014;2:4. https://doi. org/10.1186/2051-3933-2-4.

51. Avgar T, Potts JR, Lewis MA, Boyce MS. Integrated step selection analysis: bridging the gap between resource selection and animal movement. Methods Ecol Evol. 2016;7:619-30. https://doi. org/10.1111/2041-210X.12528.

52. Frair JL, Nielsen SE, Merrill EH, Lele SR, Boyce MS, Munro RHM, et al. Removing GPS collar bias in habitat selection studies. J Appl Ecol. 2004;41:201-12. https://doi.org/10.1111/j.0021-8901.2004.00902.x.

53. Ward MP, Sperry JH, Weatherhead PJ. Evaluation of automated radio telemetry for quantifying movements and home ranges of snakes. J Herpetol. 2013;47:337-45. 